\title{
Part 6
}

\section{First Results from New Facilities}




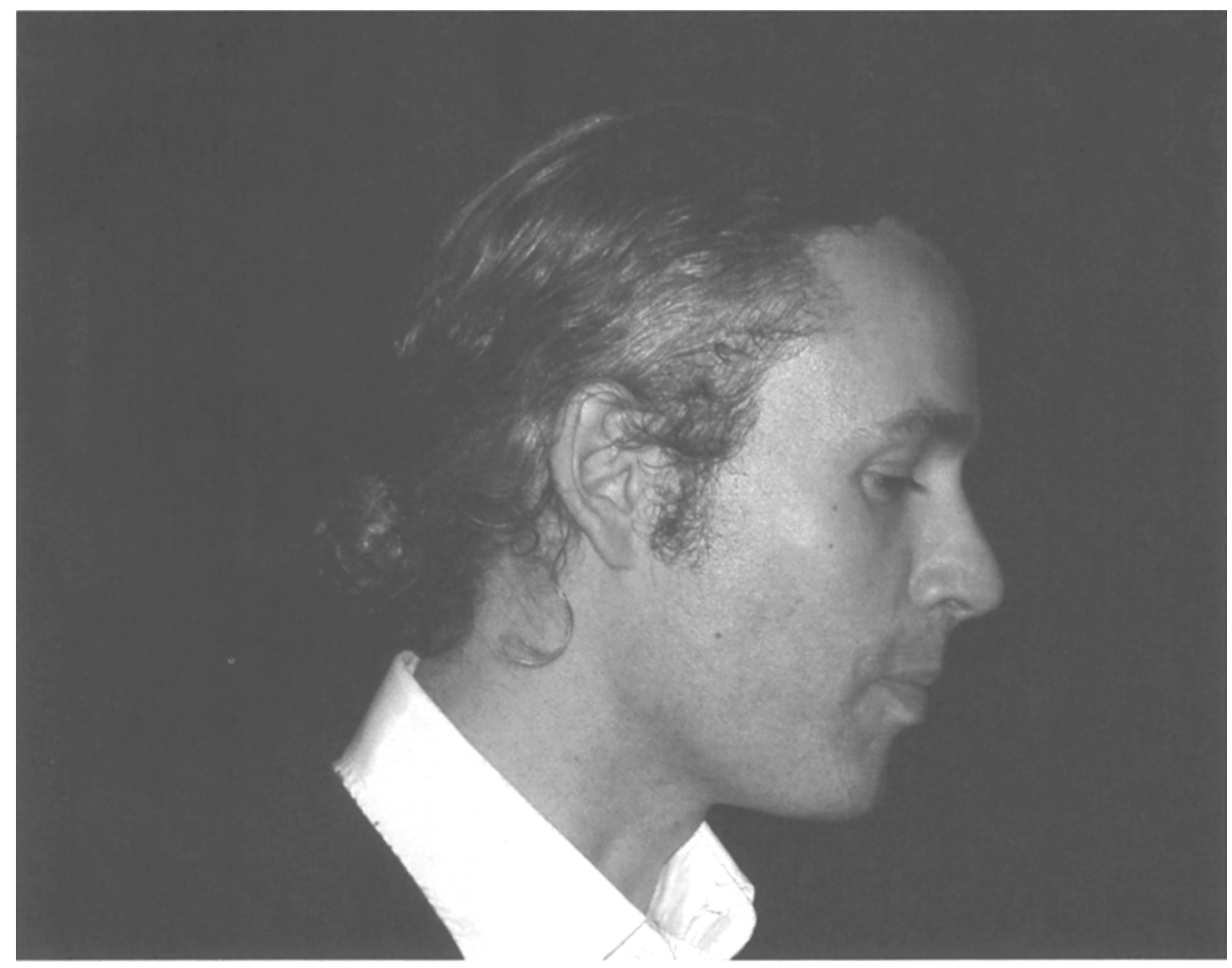

Vincent Minier 


\title{
First Results from ATCA at Millimetre Wavelengths
}

\author{
Vincent Minier \\ School of Physics, University of New South Wales, Sydney 2052, \\ Australia
}

\begin{abstract}
The newly upgraded Australia Telescope Compact Array (ATCA) at millimetre wavelengths is the first millimetre interferometer to be built in the Southern Hemisphere. The full array will be operational in 2004-2005 and will provide arcsec angular resolution at $3 \mathrm{~mm}$ and 12 $\mathrm{mm}$. This will be a unique instrument to study at high angular resolution the interstellar chemistry and more generally the star formation process, especially in the bulk of the galactic plane and in the Magellanic Clouds. The upgraded ATCA will also be an excellent tool to detect dust emission from nearby protoplanetary disks. In this paper I will present the first results from the upgraded ATCA at $3 \mathrm{~mm}$ and $12 \mathrm{~mm}$. The result review will cover the topics of massive star formation and hot molecular cores dust emission from star-forming regions and detection of protoplanetary disks.
\end{abstract}

\section{Introduction}

The Australia Telescope Compact Array (ATCA) - the radio interferometer of the Australia Telescope National Facility - has recently been upgraded to become the first millimetre interferometer in the Southern Hemisphere. ATCA, a telescope that traditionally operates at radio frequencies (i.e. wavelengths of a few $\mathrm{cm}$ to a few tens of $\mathrm{cm}$ ), will henceforth allow observations at wavelengths of $12 \mathrm{~mm}$ and $3 \mathrm{~mm}$.

To date three of the six antennas have been equipped with the 3 -mm receiver and the full array is now operational at $12 \mathrm{~mm}$. Once fully upgraded, the ATCA millimetre interferometer (ATCA-mm) will perform in the frequency ranges 16$26 \mathrm{GHz}$ and $85-110 \mathrm{GHz}$ (the 12 and $3 \mathrm{~mm}$ bands, respectively). ATCA-mm will be particularly suitable for studying processes in which molecular line emission at $12 \mathrm{~mm}$ (e.g. ammonia and water vapour masers) and $3 \mathrm{~mm}$ (e.g. methanol and silicon monoxide) are predominant. It will also be suitable to observe continuum emission (e.g. free-free emission and dust grain thermal emission).

These new instruments in the Southern Hemisphere have already attracted interest from various astronomy groups overseas (e.g. US, France, Sweden) and in Australia. To date, the main astrophysical topics studied with ATCA-mm are Star Formation Process in the Milky Way and in the Large Magellanic Cloud, Search for Protoplanetary Disks, Stellar Astrophysics (AGB stars, SNR) and Astrobiology/Exobiology. A review of the main results in Star Formation during 
2002-2003 is given below while a more general presentation of the upgraded ATCA can be found in Wong \& Melatos (2002).

\section{Pre-planetary disks around young stars}

Many young stars exhibit emission from circumstellar dust grains located in disks with properties similar to the early Solar system. Much activity is currently devoted to characterizing the physical properties of these disks to extract information on planet formation. Observations at millimetre wavelengths are especially important because the disk material beyond a few stellar radii is at temperatures from a few hundreds to a few tens of degrees Kelvin, and the physical and chemical conditions can be probed in detail in this part of the spectrum.

In 2002 using ATCA-mm, Wilner et al. (2003) observed TW Hya, the closest known classical T Tauri star, and HD 100546, a nearby Herbig Be star whose infrared spectrum shows crystalline silicates, indicative of comet-like dust. Two compact array configurations of three antennas were used, resulting in a resolution of about 2 arcsec. Detections were made toward both objects (Fig. 1) and have been reported in Wilner et al. (2003).
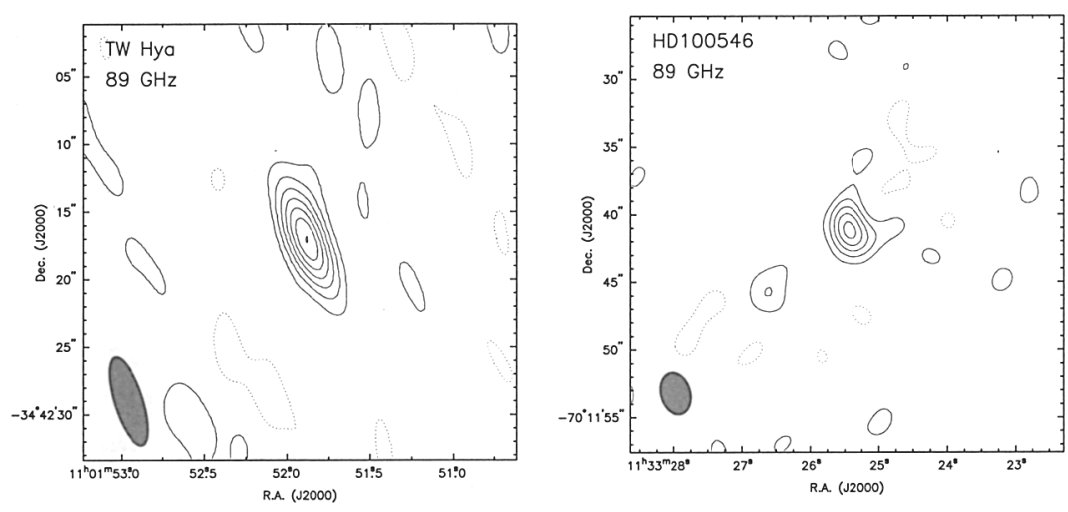

Figure 1. $\quad 89-\mathrm{GHz}$ continuum emission detected from TW Hya and HD 100546. Gaussian fits to the visibilities gives a flux of $41 \mathrm{mJy}$ for TW Hya and $36 \mathrm{mJy}$ for HD 100546. TW Hya is unresolvedwhile HD 100546 shows a hint of extended emission to the south-east. For TW Hya this flux measurement agrees well with expectations from previously reported measurements at higher and lower radio frequencies (Wilner et al. 2003). 


\section{Massive star formation in the Galactic Plane}

\subsection{Methanol masers as signposts of massive YSOs}

Interstellar masers are potentially excellent tools to identify massive YSOs because they are powerful and arise from very compact regions whose absolute positions can be measured with an accuracy of a few mas using VLBI techniques. 6.7 and $12.2 \mathrm{GHz}$ methanol masers have for instance proved to be remarkable probes in hunting down young massive stars (Minier, Conway \& Booth 2001). They are associated with sub-mm cores, colour selected IRAS sources, mid-IR sources and radio continuum sources (see Ellingsen's review, this volume), i.e signposts of massive star formation. So far, 570 methanol maser sources have been discovered via targeted surveys and unbiased surveys of the galactic plane. More interestingly, Walsh et al. (1998) have found that methanol masers are in fact generally not associated with radio sources i.e ultra-compact HII regions (UC HII regions), but rather tend to be separated from them. Preliminary work with Mopra demonstrates that methanol masers are possibly associated with hot molecular cores (HMCs). The most common molecules found in the hot, dense environment of a young massive star are for example $\mathrm{CH}_{3} \mathrm{OH}, \mathrm{NH}_{3}, \mathrm{HCN}$, $\mathrm{CH}_{3} \mathrm{CN}, \mathrm{HC}_{3} \mathrm{~N}$ and the molecular cation $\mathrm{HCO}^{+}$. Minier et al. investigated in 2002-2003, with ATCA at $3 \mathrm{~mm}$, the hot molecular core candidate associated with the methanol maser source G318.95-0.20. They used three different baseline configurations (EW214, EW352, 750A) to detect and image $\mathrm{CH}_{3} \mathrm{OH}, \mathrm{HCN}$, $\mathrm{HC}_{3} \mathrm{~N}, \mathrm{HCO}^{+}$and $\mathrm{H}^{13} \mathrm{CO}^{+}$. The $\mathrm{CH}_{3} \mathrm{OH}$ gas is confined in an unresolved core with a diameter less than $0.04 \mathrm{pc}$. The $\mathrm{HCO}^{+}$emission arises from an extended region and a central core (Fig. 2). The extended emission, rapidly resolved with increasing baselines, probably arises in a bipolar outflow. Based on these observations, the mass of the molecular core is about $15-120 \mathrm{M}_{\odot}$, confirming the presence of a massive core associated with the methanol masers.

\subsection{Probing gas conditions with methanol masers}

Methanol masers require very specific densities and temperatures to switch on. Their observation at high resolution would reveal the nature of the physical conditions in the inner part of the protostellar envelope.

Methanol masers also exist at higher frequencies in the 3-mm range. They were predicted by Sobolev et al. (1997) and were observed at 85.5, 86.6, 86.9, 107.0 and $108.8 \mathrm{GHz}$ (e.g. Cragg et al. 2001; Minier \& Booth 2002). In the southern hemisphere G345.01+1.79, a massive star-forming region, exhibits strong methanol emission at 6.7, 12.2, 85.5, 86.6, 86.9, 107.0, 108.8 and 156.6 $\mathrm{GHz}$. The millimetre emission lines have been interpreted as maser features based on their narrow line-widths, their velocity coincidence with the 6.7 and $12.2-\mathrm{GHz}$ maser features and their LSR velocities distinct from that of the quasi-thermal emission seen at $108.8 \mathrm{GHz}$ (Cragg et al. 2001). Ellingsen et al. (2003) have recently combined the multi-frequency maser observations with the Sobolev-Deguchi maser model and proposed to constrain density, dust and gas temperatures of the masing medium.

Minier et al. observed 85.5 and 86.9-GHz methanol masers in G345.01+1.79 with ATCA-mm in October 2002. Three antennas were used in the $750 \mathrm{~A}$ configuration. 85.5 and $86.9-\mathrm{GHz}$ masers were easily detected at the same velocity 

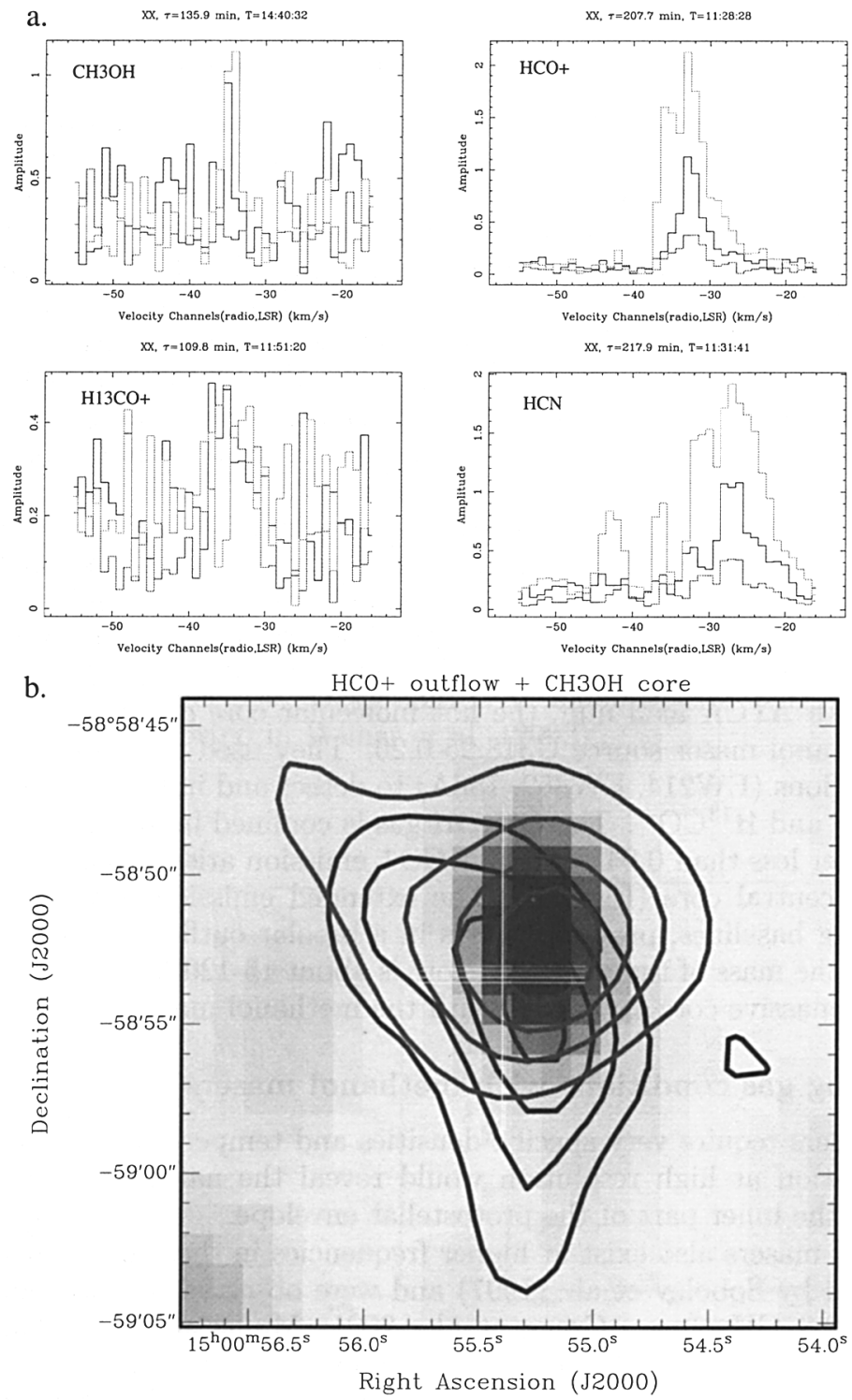

Figure 2. G318.95-0.20. a. Molecular line spectra taken on three ATCA baselines. $\mathrm{HCO}^{+}$and $\mathrm{HCN}$ emission is resolved with increasing baselines, while $\mathrm{CH}_{3} \mathrm{OH}$ intensity remains constant. b. Images of the thermal methanol emission (colour scale) and $\mathrm{HCO}^{+}$emission (blue and red contours). The white crosses represent the methanol masers that coincide with a massive $\left(15-120 \mathrm{M}_{\odot}\right)$ molecular core. The methanol core is unresolved while the $\mathrm{HCO}^{+}$emission is divided in blue- and red-shifted components. 
as the 6.7 and $12.2-\mathrm{GHz}$ masers (Fig. 3). Surprisingly, the 3-mm masers coincide in velocity and position with the $\mathrm{cm}$ masers, and hence coincide in space in G345.01+1.79. This is only possible for a narrow range of physical conditions according to the maser model (Fig. 3). Figure 3 suggests that the environment of the masing medium in G345.01+1.79 contains warm dust $(250 \mathrm{~K})$, cool gas $(40 \mathrm{~K})$ and a large methanol column-density.

\subsection{Class I methanol masers, $\mathrm{NH}_{3}$ in Orion and $\mathrm{H}_{2} \mathrm{O}$ masers}

ATCA will also be a powerful instrument to explore the southern sky using $25 \mathrm{GHz}$ methanol maser (e.g. observations by Voronkov et al.), $22 \mathrm{GHz}$ water masers and ammonia emission near $24 \mathrm{GHz}$. First detections of these lines are presented in Fig. 4.

\subsection{Deuterated ammonia in NGC6334}

Abundances of deuterated molecules can be enhanced by factors of 100 or more by isotropic fractionation. This is especially likely in cold molecular clouds, due to energy barriers for some of the key ion-molecule reactions involved in the formation and destruction of deuterated molecules (Watson 1974).

Deuterated ammonia was detected at $85.9 \mathrm{GHz}$ in the NGC6334 region by Wong, Minier \& Forster at the location of the brightest ammonia emitter in the sky, the source known as I(N). NGC6334:I(N) is known as a massive protostar candidate (Sandell 2000) with no radio continuum emission and with strong $\mathrm{mm}$ continuum emission. This is confirmed by the continuum maps at $12 \mathrm{~mm}$ and $3 \mathrm{~mm}$. No emission is detected at $12 \mathrm{~mm}$ while a strong $3-\mathrm{mm}$ continuum emission is seen at the location of $\mathrm{I}(\mathrm{N})$ (Fig. 4). At high angular resolution, NGC6334:I(N) is a cold core without ionised gas detectable and possibly harbours a high-mass protostar.

\section{Massive star formation in the LMC}

ATCA at 3 and $12 \mathrm{~mm}$ is also a unique instrument to study molecular gas emission in the LMC. Observations of molecular gas by Wong et al. in N113 (see Wong's paper, this volume) as well as a search for new water masers (Beasley et al.) have been undertaken in 2002-2003.

\section{Conclusions}

In 2002-2003, 43 projects have been carried out with the upgraded ATCA at millimetre wavelengths. 27 projects were directly related to star formation. The 12 -mm system is fully operational while the $3-\mathrm{mm}$ upgrade is expected to be complete in 2004-2005. The ATCA-mm is ongoing continuous upgrade and the observers are invited to visit the ATCA-mm web page for information on the current status of the $\mathrm{mm}$ receivers (www.atnf.csiro.au/observers/docs $/ 3 \mathrm{~mm} /$ ).

Acknowledgments. The author thanks all the ATCA observers who have kindly provided their results in advance of publication as well as Tony Wong, Michael Burton and the ATCA staff for their commitment to the development of a millimetre interferometer in Australia. 

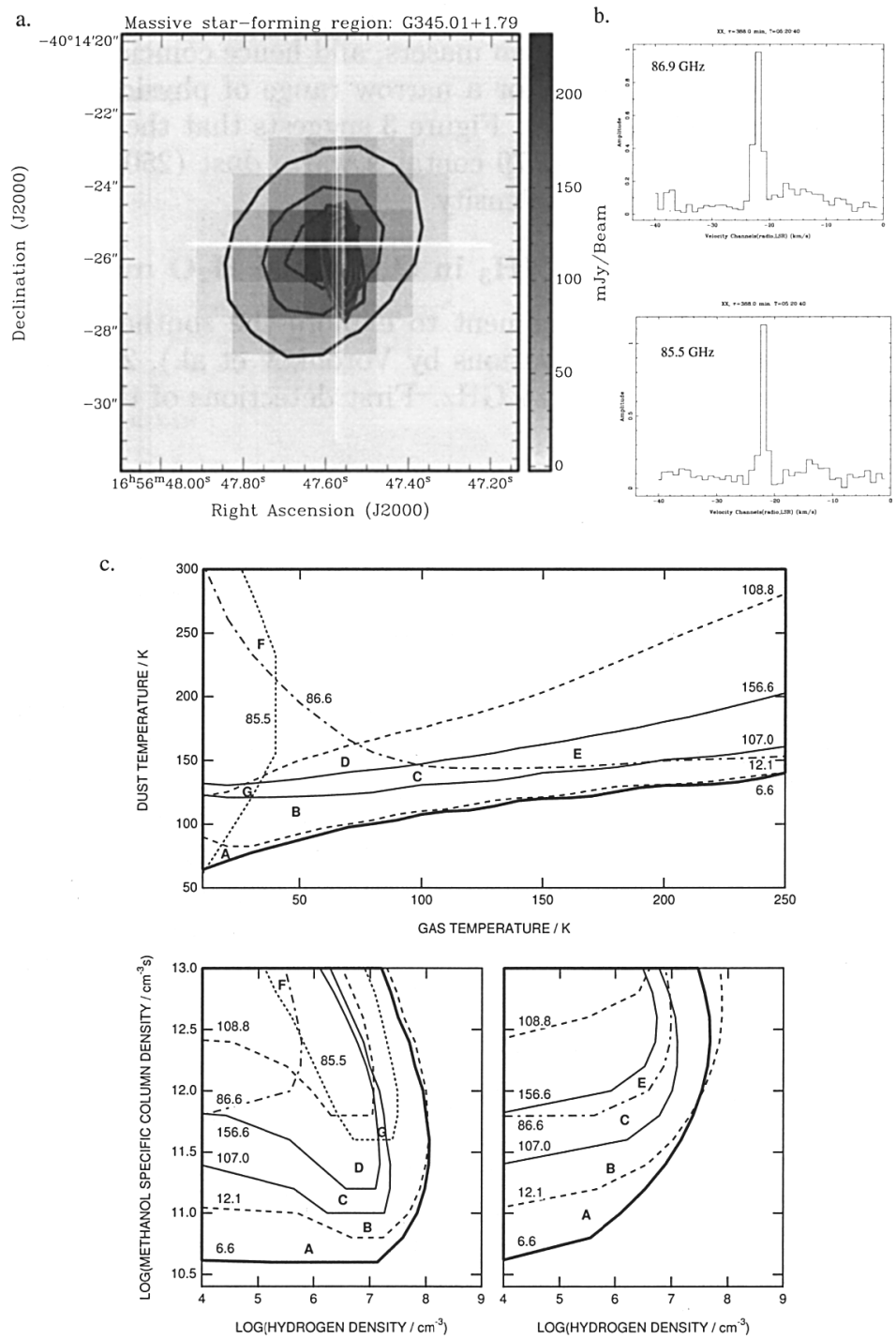

Figure 3. Methanol masers in G345.01+1.79. a. Positions of the 6.7 and $12.2-\mathrm{GHz}$ masers (white crosses) and of the 85.5 and $86.9-\mathrm{GHz}$ methanol masers (blue and red contours) overlaid on the radio continuum emission (colour scale and grey contours). b. ATCA 3-mm maser spectra. c. Modelling of the maser suitable conditions (Ellingsen et al. 2003). The contour diagrams show where methanol masers become active as function of density and temperature. Contours are labelled with the maser frequency in $\mathrm{GHz}$ and represent a brightness temperature threshold of $10^{6} \mathrm{~K}$. For instance, all masers are quenched for hydrogen densities greater than $10^{8} \mathrm{~cm}^{-3}$. G345.01+1.79 corresponds to point $\mathrm{F}$ in the diagram for which all transitions could be detected. 


\section{References}

Cragg, D.M., Sobolev, A.M., Ellingsen, S.P., et al. 2001, MNRAS, 323, 939

Ellingsen, S.P., Cragg, D.M., Minier, V., Muller, E., Godfrey, P.D. 2003, MNRAS, 344,73

Forster, J.R., Whiteoak, J.B., Gardner, F.F., Peters, W.L., \& Kuiper, T.B.H. 1987, PASA, 7, 189

Minier, V., Conway, J.E., \& Booth, R.S. 2001, A\&A, 369, 278

Minier, V., \& Booth, R.S. 2002, A\&A 387, 179

Sandell, G. 2000, A\&A, 358, 242

Sobolev, A.M,, Cragg, D.M., \& Godfrey, P.D. 1997, MNRAS, 288, L39

Walsh, A.J, Burton, M.G., Hyland, A.R, \& Robinson, G. 1998, MNRAS, 301, 640

Watson, W.D. 1974, ApJ, 188, 35

Wilner, D.J., Bourke, T.L., Wright, C.M., et al. 2003, ApJ, in press

Wong, T., \& Melatos, A. 2002, PASA, 19, 475
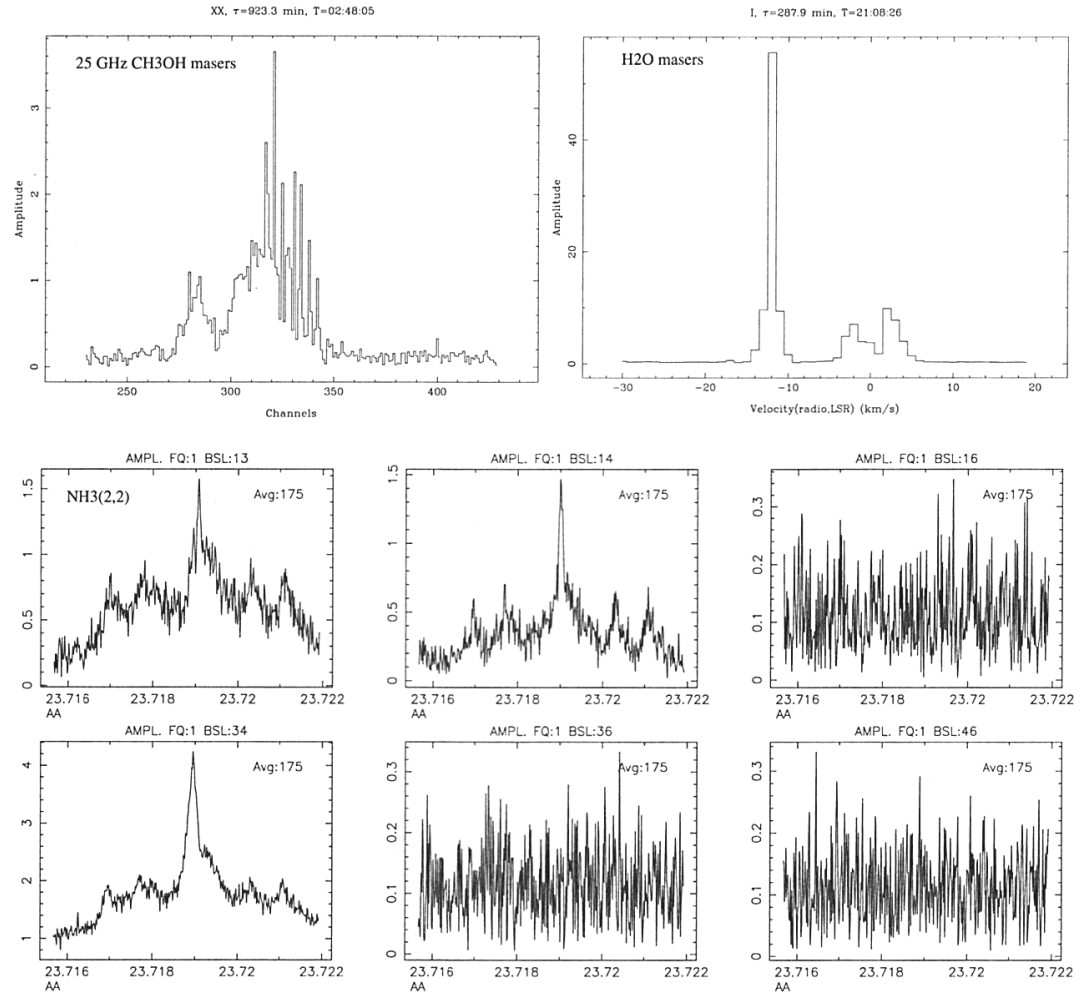

Figure 4. $25 \mathrm{GHz}$ methanol masers in Orion KL (observations by Voronkov et al. in 2003), water masers in $\mathrm{G} 345$ and $\mathrm{NH}_{3}(2,2)$ emission in Orion KL (first light in April 2003) 
a.

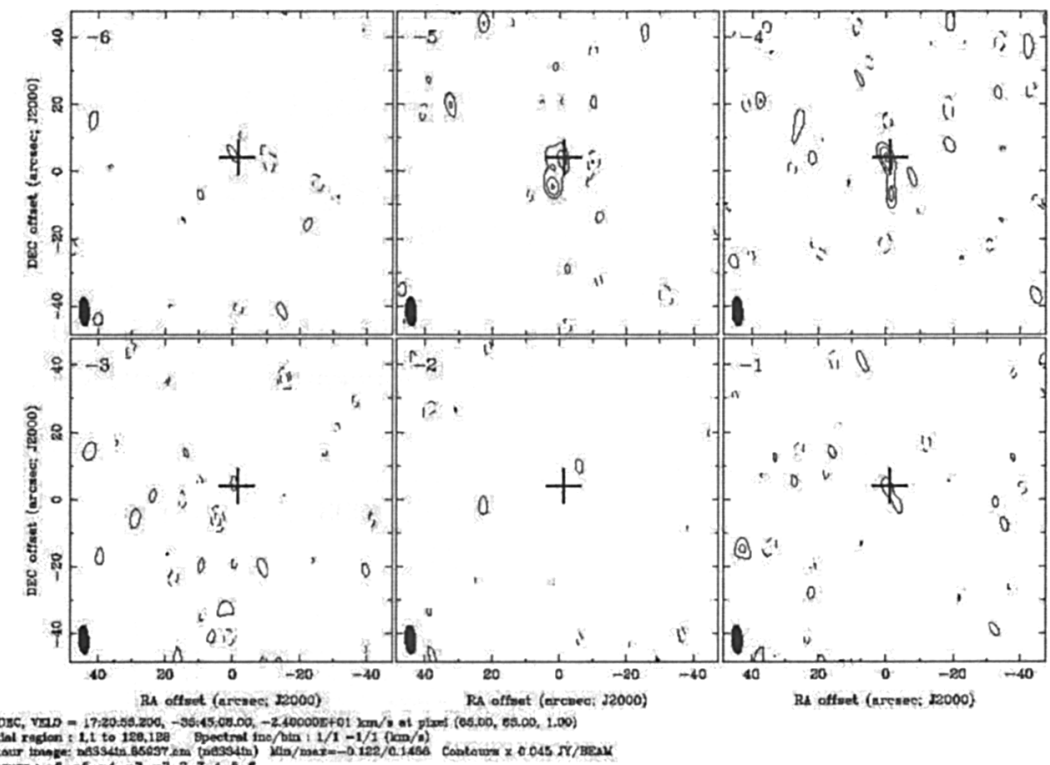

b.

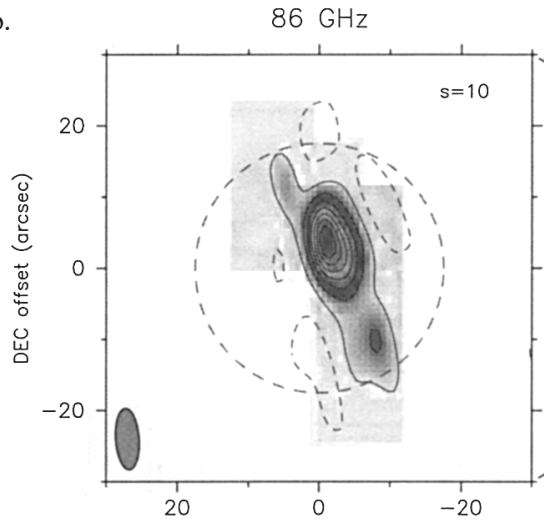

$86 \mathrm{GHz}$

$19 \mathrm{GHz}$

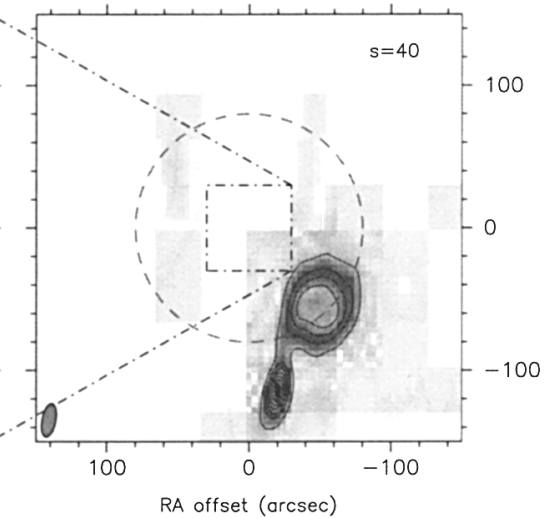

Pointing .centre 17:20:55.2, -35:45:08 (J2000)

Contour levels $(1,3,5,7, \ldots) \times \mathrm{s} \mathrm{mJy} / \mathrm{bm}$, where $\mathrm{s}$ is given above

Observations conducted 18 \& 20 April 2003

Figure 5. NGC6334. a. Deuterated ammonia channel maps taken with ATCA at $85.9 \mathrm{GHz}$ in NGC6334:I(N). The velocity is given in each channel map. b. Continuum maps at 19 and $86 \mathrm{GHz}$. Observations were made by Wong, Minier \& Forster in 2003. 